\title{
Radiation-induced sterility for pupal and adult stages of the malaria mosquito Anopheles arabiensis Michelle EH Helinski ${ }^{1}$, Andrew G Parker ${ }^{1}$ and Bart GJ Knols*1,2
}

\author{
Address: ${ }^{1}$ Entomology Unit, FAO/IAEA Agriculture and Biotechnology Laboratory, International Atomic Energy Agency (IAEA), A-2444 \\ Seibersdorf, Austria and ${ }^{2}$ Laboratory of Entomology, Wageningen University and Research Center, P.O. Box 8031, 6700 EH Wageningen, The \\ Netherlands \\ Email: Michelle EH Helinski - m.helinski@iaea.org; Andrew G Parker - a.parker@iaea.org; Bart GJ Knols* - b.knols@iaea.org \\ * Corresponding author
}

Published: 15 May 2006

Malaria Journal 2006, 5:41 doi:I0.1 186/I475-2875-5-4I

This article is available from: http://www.malariajournal.com/content/5/I/4I

(C) 2006 Helinski et al; licensee BioMed Central Ltd.

This is an Open Access article distributed under the terms of the Creative Commons Attribution License (http://creativecommons.org/licenses/by/2.0), which permits unrestricted use, distribution, and reproduction in any medium, provided the original work is properly cited.
Received: 23 February 2006

Accepted: 15 May 2006

\begin{abstract}
Background: In the context of the Sterile Insect Technique (SIT), radiation-induced sterility in the malaria mosquito Anopheles arabiensis Patton (Diptera: Culicidae) was studied. Male mosquitoes were exposed to gamma rays in the pupal or adult stage and dose-sterility curves were determined.

Methods: Pupae were irradiated shortly before emergence (at 22-26 hrs of age), and adults $<24$ hrs post emergence. Doses tested ranged between 0 and $100 \mathrm{~Gy}$. The effects of irradiation on adult emergence, male survival, induced sterility and insemination capability were evaluated. Emergence and insemination data were analysed using independent $t$-tests against the control. Correlation analyses were performed for insemination rate and dose and insemination and fecundity. Male survival was analysed using Kaplan-Meier survival analyses. Finally, the calculated residual fertility values were inverse-normal transformed and linear regression analyses performed.

Results: Irradiation of pupae, for all doses tested, had no effect on adult emergence. Survival curves of males irradiated as pupae or adults were similar or even slightly higher than nonirradiated males. Overall, adults appeared to be slightly more susceptible to irradiation, although no significant differences for individual doses were observed. In the pupal stage, a significant negative correlation was found between insemination and dose, but the correlation-coefficient was associated with less than $25 \%$ of the total variation. A review of the literature indicated that An. arabiensis is more radiation resistant than other anopheline mosquitoes.

Conclusion: The optimal dose for male insects to be released in an SIT programme depends on their level of sterility and competitiveness. The use of semi-sterilizing doses to produce more competitive insects is discussed. The most convenient developmental stage for mosquito irradiation on a mass-scale are pupae, but pupal irradiation resulted in a lower insemination rate at the highest dose compared to adult irradiation. On the basis of this study, a suitable dose range that includes semi-sterilizing doses is identified to initiate competitiveness experiments for males irradiated at both developmental stages.
\end{abstract}

\section{Background}

In the $21^{\text {st }}$ century, anopheline mosquitoes remain the most deadly insects in the world. Malaria is still widely spread; it is estimated that currently 3.2 billion people live 
in areas at risk of malaria transmission [1]. Estimated economic growth reduction in endemically affected countries is high, and contemporary control methods are not always effective due in part to widespread resistance of the mosquitoes to insecticides and Plasmodium parasites to commonly used drugs. The Sterile Insect Technique (SIT), successfully applied against a number of pest species [2], has been evaluated against Anopheles albimanus Wiedemann in the 1970 s with encouraging results $[3,4]$. Over the last years, a renewed interest in SIT for malaria vectors has led to a 5-year feasibility study to investigate all aspects of an SIT programme including sexing, mass production, sterilisation, and release methodology $[5,6]$. The project initially focuses on the African malaria vector Anopheles arabiensis Patton.

The SIT relies on the sterilisation of insects by chemosterilisation $[4,7,8]$, irradiation [2] or modern biotechnological approaches [9-11]. Modern biotechnological approaches based on transgenic organisms are promising but at an early stage of development and no legal framework yet exists to facilitate the introduction of such organisms in the wild $[12,13]$. Sterilisation by irradiation or chemosterilants has not been researched extensively for the last 30 years with mosquitoes. Promising results were obtained with chemosterilants in terms of the level of sterility induced and competitiveness present [8] but these have the disadvantage of being mutagenic agents. They thus present a potential hazard to humans during the treatment process and non-target organisms if residues persist in released individuals [8]. Even though the actual amount of residue released in the environment was in fact very low due to careful rinsing of the pupae [14], concerns raised about the possible negative effects on the environment if large numbers of treated insects were to be released $[15,16]$ resulted in a disuse of chemosterilants for mosquito control. Although it would be worthwhile to identify additional compounds with chemosterilant properties, it remains doubtful if the currently available ones will be acceptable for use in future genetic control programmes.

Sterilisation by irradiation remains the most practical way to sterilise the insects at present, and it has been argued that radiation sterilisation should also be used to introduce the first transgenic organisms in the wild [3]. Determining the optimal dose range for an SIT programme depends on the level of sterility induced and the competitiveness of the irradiated males. A low dose may result in insufficiently sterilised males, whereas a high dose may undermine the insect's ability to compete with wild con-specifics and may thus reduce the overall impact of the release.

In the context of SIT, anopheline irradiation has been performed on a number of species and dose-response curves have been published for An. albimanus [17], An. pharoensis Theobald $[18,19]$ and An. stephensi Liston $[7,20]$. An. arabiensis has been studied in the light of genetic sexing systems [21] and small-scale irradiation studies [22] but no dose-response curve exists. Previous work has indicated that substantial inter-species variation in radiation sensitivity is present [22], justifying the need for a dose-sterility curve for An. arabiensis. In mosquitoes, both the pupal and the adult stage can be irradiated. Pupal irradiation is easier to perform, but there is evidence of a reduced competitiveness when male pupae are irradiated at high doses compared to adult irradiation [23]. The objective of this study was to determine the dose-sterility curves for the pupal and adult stages of male An. arabiensis and define a suitable dose range to initiate competitiveness experiments.

\section{Methods \\ Mosquitoes}

The mosquito strain used is the "KGB" strain of An. arabiensis. The strain originates from Zimbabwe and has been colonized since 1975 (Courtesy of MR4, CDC Atlanta, USA). All mosquitoes used in the experiments were reared at a density of $\sim 750$ larvae per tray $(30 \times 40 \mathrm{~cm})$ containing \pm 2 liter of deionized water (water depth $2 \mathrm{~cm}$ ). Heating mats were used to maintain the water temperature at $28^{\circ} \mathrm{C}$. Larvae were fed a diet of fish-food (Aquaricare ${ }^{\circledast}$ ) daily ( $0.3 \mathrm{mg} / \mathrm{larva})$ that was powdered and passed through a $224 \mu \mathrm{m}$ mesh sieve. Adults were maintained in the insectary at $28^{\circ} \mathrm{C}$ and $80 \% \mathrm{RH}$ and all experiments were conducted in standard $30 \mathrm{~cm}$ cubic cages. The light regime was L10:D12 with a one hour simulated dusk and dawn period. Adult cages were continuously supplied with $10 \%$ sucrose solution $[\mathrm{w} / \mathrm{v}]$.

\section{Irradiation procedure}

Insects were exposed to gamma rays generated by a cobalt60 source (Gammacell 220, MDS Nordion, Ottawa, Canada) with a dose rate of ca $16 \mathrm{~Gy} / \mathrm{min}$. Insects were concentrated in the centre of the chamber to maximise dose uniformity within the batch (Fig. 1). A dosimetry system was used to measure the dose received by the batch based on the Gafchromic HD-810 film (International Specialty Products, NJ, USA) [24]. Three dosimeters were included with each batch of insects and read after irradiation with a Radiachromic ${ }^{\circledast}$ reader (Far West Technology, Inc., California, USA).

\section{Experimental set-up}

Experiments were performed in series. Each series included a number of different irradiation doses and a control (Table 1). The experimental mosquitoes in each series originated from the same batch of material and were randomly distributed to treatment. Mosquitoes in the control group underwent exactly the same handling stages as the experimental mosquitoes, except the actual 
irradiation itself. Within each series, order of irradiation was assigned randomly. We covered a spectrum of doses that ranged from a control (no irradiation) to a dose previously shown to yield almost complete sterility $[7,25]$. These were $0,25,50,60,70,80$, and 100 Gy for the pupal and adult experiments. In the pupal experiments, two additional doses of 35 and 45 Gy were included. Each dose was replicated twice, and the doses between 50 and 80 were replicated three times.

\section{Parameters measured}

\section{Emergence}

Emergence from irradiated pupae was scored for both sexes, as pupae were not sexed prior to irradiation. Only mosquitoes that successfully emerged were positively scored; semi-emerged adults and dead adults were scored as non-emerged.

\section{Longevity}

The longevity of the irradiated mosquitoes was determined by the removal and counting of dead mosquitoes at $24 \mathrm{hr}$ intervals (except during weekends, when 48-72 $\mathrm{hr}$ intervals were used). Cages were discarded when at least two-third of all the males had died.

\section{Sterility assessment}

Levels of sterility were observed by mating the irradiated males with non-irradiated virgin females. To ensure virginity of females, pupae were placed in individual tubes prior to emergence. Mates were introduced into experimental cages in a 1:1 ratio. For experiments with pupae, mates were introduced into the cages the day after irradiation when the males had emerged. For the adult experiments, mates were introduced after the irradiation on the same day. Mosquitoes were fed on the forearm of a volunteer for 10 min twice on consecutive days, between day 2 and 5 after introduction of the females (with the exception of series 1 in the adult stage when mosquitoes were fed on a membrane filled with human blood). Egg laying occurred en masse in the cage. For each cage, one egg bowl filled with water and lined with wet filter paper was offered for five nights starting two days after the first blood meal. Daily, or at $48 \mathrm{hr}$ intervals, eggs were removed and counted. For hatch rate determination, the eggs were thoroughly mixed, and random samples of eggs were placed in larger trays to allow hatching. Trays from control cages were filled with ca. 200 eggs/tray, and more eggs were placed in one tray as the doses increased due to higher sterility levels of the eggs. Eggs were checked for

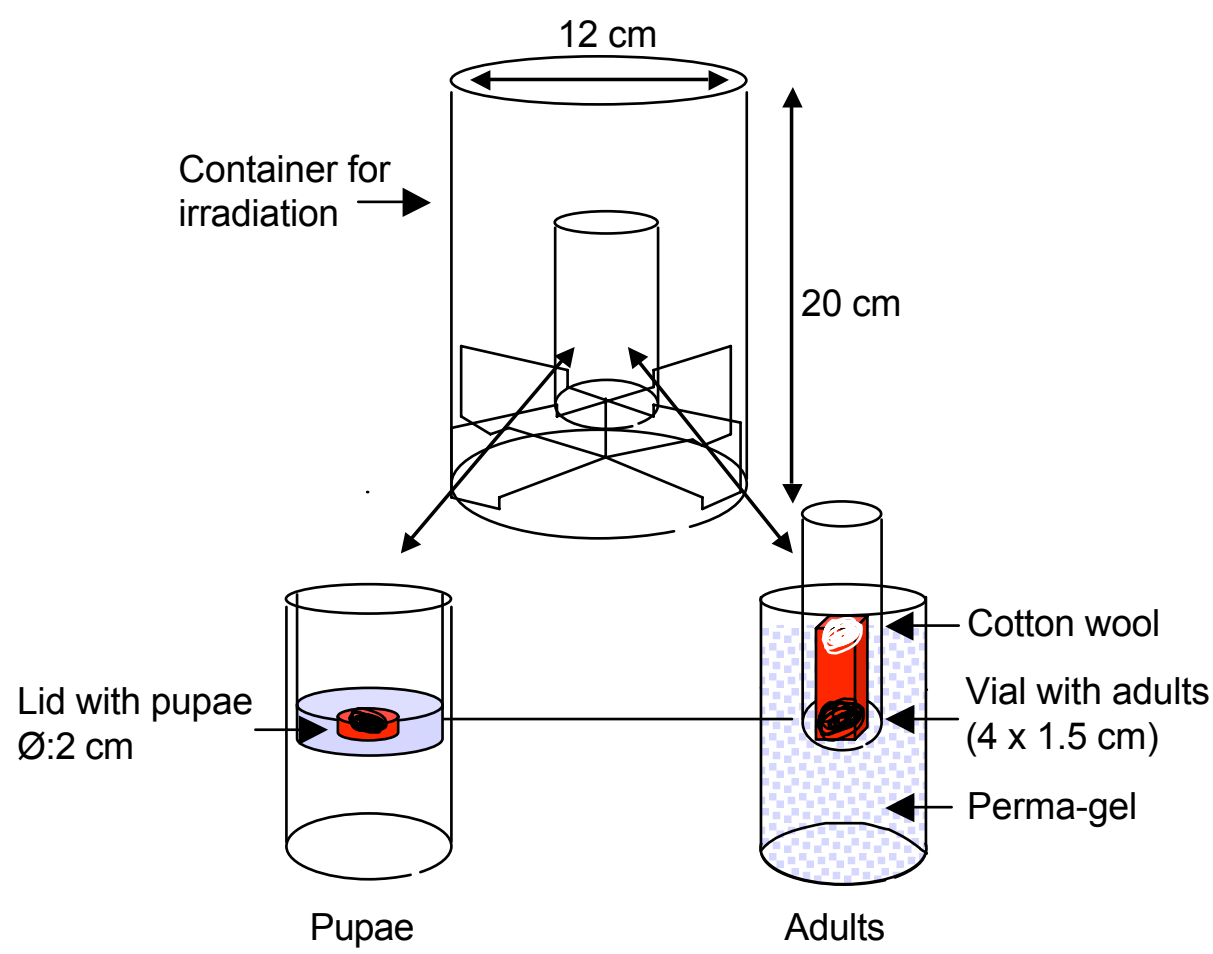

\section{Figure I}

Insects were irradiated in a confined space in the centre of the irradiation chamber to minimize maximise dose uniformity within the batch. Devices used for the irradiation of pupae and adults are shown. 
Table I: Effect of different irradiation doses on median ( \pm SE) survival times of An. arabiensis males. Log-rank tests compared treatments with the control. Survival time was estimated from Kaplan-Meier survival analysis (details see text).

\begin{tabular}{|c|c|c|c|c|c|c|c|c|c|}
\hline Pupae Series & Dose (Gy) & $\mathrm{n}$ & Median survival time & Log-Rank & Adult Series & Dose (Gy) & $\mathrm{n}$ & Median survival time & Log-Rank \\
\hline \multirow[t]{3}{*}{1} & 0 & 31 & $13 \pm 0.6$ & $\mathrm{Na}$ & 1 & 0 & 57 & $7 \pm 0.8$ & $\mathrm{Na}$ \\
\hline & 25 & 35 & $7 \pm 1.7$ & $14.66 * *$ & & 60 & 55 & $10 \pm 0.8$ & 1.59 \\
\hline & 35 & 27 & $11 \pm 1.0$ & $6.09 *$ & & 70 & 56 & $10 \pm 0.6$ & $5.10^{*}$ \\
\hline \multirow[t]{3}{*}{2} & 0 & 56 & $9 \pm 1.1$ & $\mathrm{Na}$ & & 80 & 52 & $10 \pm 0.9$ & 0.04 \\
\hline & 50 & 48 & $10 \pm 0.2$ & 0.84 & 2 & 0 & 54 & $8 \pm 0.8$ & $\mathrm{Na}$ \\
\hline & 70 & 55 & $6 \pm 2.9$ & 0.10 & & 50 & 59 & $10 \pm 1.1$ & 0.07 \\
\hline \multirow[t]{3}{*}{3} & 0 & 48 & $11 \pm 1.5$ & $\mathrm{Na}$ & & 60 & 51 & $10 \pm 0.9$ & 0.16 \\
\hline & 80 & 51 & $13 \pm 2.3$ & $4.93^{*}$ & & 70 & 56 & $7 \pm 1.2$ & 0.11 \\
\hline & 100 & 61 & $11 \pm 1.7$ & 1.63 & & 80 & 50 & $10 \pm 1.3$ & 2.60 \\
\hline \multirow[t]{4}{*}{4} & 0 & 46 & $6 \pm 1.5$ & $\mathrm{Na}$ & & 100 & 55 & $10 \pm 0.9$ & 0.00 \\
\hline & 35 & 54 & $13 \pm 0.8$ & 2.70 & 3 & 0 & 49 & $10 \pm 1.9$ & $\mathrm{Na}$ \\
\hline & 45 & 58 & $\mathrm{II} \pm 0.5$ & 1.08 & & 25 & 48 & $10 \pm 1.7$ & 0.84 \\
\hline & 50 & 53 & $I I \pm I . I$ & 0.58 & & 50 & 45 & $10 \pm 1.1$ & 0.05 \\
\hline \multirow[t]{4}{*}{5} & 0 & 32 & $10 \pm 2.2$ & $\mathrm{Na}$ & & 100 & 47 & $10 \pm 1.7$ & 0.44 \\
\hline & 60 & 45 & $10 \pm 0.8$ & 0.34 & 4 & 0 & 48 & $6 \pm 2.7$ & $\mathrm{Na}$ \\
\hline & 70 & 35 & $10 \pm 2.1$ & 1.29 & & 25 & 48 & $15 \pm 2.4$ & 1.06 \\
\hline & 80 & 35 & $12 \pm 0.9$ & 0.77 & & 50 & 48 & $12 \pm 2.2$ & 1.79 \\
\hline \multirow[t]{6}{*}{6} & 0 & 44 & $7 \pm 1.2$ & $\mathrm{Na}$ & & 60 & 48 & $11 \pm 1.5$ & 0.29 \\
\hline & 45 & 42 & $10 \pm 2.2$ & 3.74 & & 70 & 46 & $11 \pm 1.2$ & 0.59 \\
\hline & 60 & 42 & $4 \pm 1.3$ & 0.19 & & 80 & 49 & $11 \pm 4.2$ & 0.00 \\
\hline & 70 & 41 & $10 \pm 1.6$ & 2.32 & & & & & \\
\hline & 80 & 41 & $10 \pm 1.0$ & 2.94 & & & & & \\
\hline & 100 & 43 & $10 \pm 1.2$ & 0.70 & & & & & \\
\hline \multirow[t]{6}{*}{7} & 0 & 44 & $10 \pm 1.4$ & $\mathrm{Na}$ & & & & & \\
\hline & 25 & 45 & $12 \pm 1.7$ & $10.63^{* *}$ & & & & & \\
\hline & 45 & 42 & $12 \pm 0.6$ & $11.57^{* *}$ & & & & & \\
\hline & 50 & 41 & $10 \pm 0.3$ & 0.07 & & & & & \\
\hline & 60 & 45 & $\mathrm{II} \pm 0.7$ & $5.73 *$ & & & & & \\
\hline & 50 & 42 & $12 \pm 0.9$ & $8.10^{* * *}$ & & & & & \\
\hline
\end{tabular}

Na: not applicable; *: $\mathrm{P}<0.05 ; * *: \mathrm{P}<0.0$ I

their hatchability by counting and removing L1 larvae daily for seven consecutive days from the trays.

For each day of egg collection, all eggs or a sample of all eggs were used to determine hatch rates. Since eggs were thoroughly mixed, the sample can be regarded as representative of the total amount laid for that day and treatment, therefore hatch rates were weighted to the total number of eggs collected for that day. For each treatment, an average value was obtained per series by weighting the data for all egg collection days. The residual fertility was calculated as a percentage of the control fertility of each series and subtracted from 100\% (Abbott's formula [26]) to give a value for radiation-induced sterility.

Fecundity (the average number of eggs laid per female) was calculated by dividing the number of eggs laid per night by the number of females alive at the start of that night. A value for each treatment per series was obtained by the sum of all egg laying nights and data were pooled per treatment for both stages.

\section{Insemination}

The proportion of females inseminated by irradiated males was extrapolated from dissections of a sub-sample of females tested. After egg laying, a random selection of females was taken from the cages and their spermatheca dissected to examine whether these had been inseminated. The presence of spermatozoa was confirmed using a compound microscope at $400 \times$ magnification.

\section{Collection and irradiation of experimental mosquitoes Pupae}

Pupae were collected the day before irradiation at $3 \mathrm{pm}$ from trays that had been cleared of all their pupae before 9 am that day to ensure equal age of the pupae. At $11 \mathrm{am}$ the next day, the pupae, aged 22-26 hrs, were irradiated. Pupae were irradiated in a small plastic lid filled with water (Fig. 1). For each dose, 100 pupae were irradiated at once. After irradiation, individual pupae were put in small vials and left overnight for emergence. The following morning, males were transferred to the cages according to treatment. 
Adults

Irradiation of adults was performed on specimens $<24$ hrs of age. Pupae were collected from trays and allowed to emerge overnight in a standard cage. The following morning, the mosquitoes were separated by sex and males were placed in a small holding cup prior to irradiation and sugar water was offered on cotton wool. Fifty adult males were irradiated for each dose. Adults were irradiated in a modified version of the pupal irradiation device (Fig. 1). Perma-gel ${ }^{\circledast}$ from an ice-pack (Ice-pak ${ }^{\mathrm{TM}}$, Cryopak Industries Inc., Canada) surrounded a tube in which a small vial was placed that contained the adults. The irradiation device was placed at $\sim 4^{\circ} \mathrm{C}$ prior to irradiation to cool the gel and immobilize the adults during the irradiation. Adults were immobilized in an ice-box $\left(\sim 4^{\circ} \mathrm{C}\right)$ for $5 \mathrm{~min}$ before irradiation and transferred to the vial. The opening of the vial was closed with some cotton wool and placed in the irradiation device. After irradiation, the vial was opened inside the cage and the adults were left to recover and disperse.

\section{Statistical analysis}

Data was analysed using the following variables: treatment (dose: 0, 25, 35, 45, 50, 60, 70, 80, $100 \mathrm{Gy)}$, series $(1-7)$, and stage (pupae or adults). Unless stated otherwise, for both stages, data from the same treatments between different series were pooled to get an average value per treatment. Proportional values for adult emergence and insemination were arcsine-square-root-transformed to achieve normal distribution. Independent ttests were used to determine significant differences between the mean values of treated groups and the control. In addition, correlation analyses (Pearson correlation coefficient) between dose and insemination rate, and between fecundity and insemination rate were performed for both stages.

Survival curves were analysed using Kaplan-Meier survival analyses. The obtained survival curves were compared to the control using Mantel-Cox log-rank tests.

General Linear Models (GLM) were used to observe differences in fecundity between treatments for the two stages, and to compare induced sterility levels between stages.

The calculated residual fertility values were inverse-normal transformed to yield normal equivalent deviates (N.E.D.) and irradiation doses were $\log _{10}$ transformed to obtain a linear relationship between dose and residual fertility. A standard linear regression analysis was performed for each stage with $\log _{10}$ (Dose/Gy) as the independent and N.E.D. as the dependent variable.

All two-sided tests were performed using SPSS version 12 (SPSS Inc., Chicago, IL).

\section{Results}

Dosimetry confirmed that all doses delivered lay within a $5 \%$ error range.

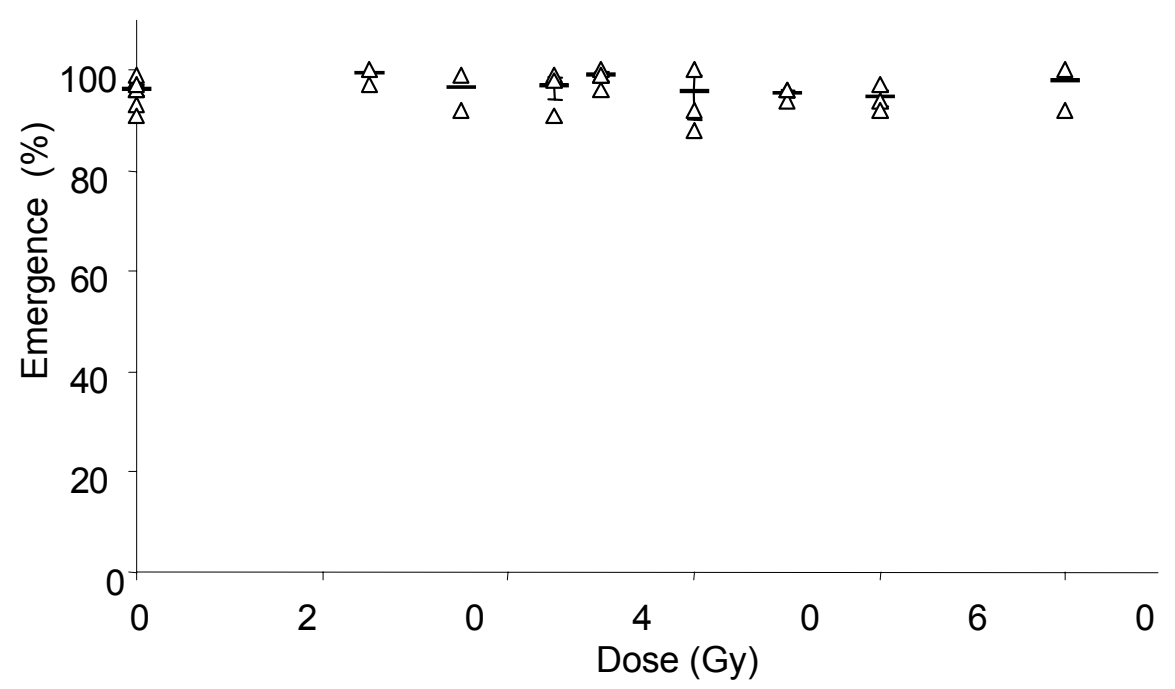

\section{Figure 2}

Adult emergence for the different treatments. Triangles indicate individual values; detransformed means $( \pm S E$, if $n>2)$ are indicated by a horizontal line. 
Table 2: Mean ( \pm SE) induced sterility levels and mean $( \pm$ SE) fecundity of females mated with An. arabiensis males irradiated in the pupal or adult stage. A range is indicated where number of replicates is $<2$ (n: total number of females alive at onset of egg-laying period; Nd: not done).

\begin{tabular}{|c|c|c|c|c|c|c|c|c|}
\hline \multirow[t]{2}{*}{ Treatment Dose (Gy) } & \multicolumn{4}{|c|}{ Pupal irradiation } & \multicolumn{4}{|c|}{ Adult irradiation } \\
\hline & Replicates & Induced sterility & Fecundity & $\mathrm{n}$ & Replicates & Induced sterility & Fecundity & $\mathrm{n}$ \\
\hline 0 & 7 & 0.0 & $45 \pm 4.9$ & 266 & 4 & 0.0 & $42 \pm 11.6$ & 169 \\
\hline 25 & 2 & $35.4(32.3-38.5)$ & $32(25-38)$ & 56 & 2 & $38.9(38.7-39.0)$ & $69(62-76)$ & 84 \\
\hline 35 & 2 & $44.8(35.7-53.8)$ & $52(49-54)$ & 59 & $\mathrm{Nd}$ & & & \\
\hline 45 & 3 & $68.3 \pm 6.9$ & $42 \pm 5.7$ & 125 & $\mathrm{Nd}$ & & & \\
\hline 50 & 4 & $76.0 \pm 4.6$ & $34 \pm 5.0$ & 178 & 3 & $71.7 \pm 5.8$ & $46 \pm 11.0$ & 135 \\
\hline 60 & 3 & $78.6 \pm 5.7$ & $39 \pm 8.6$ & 104 & 3 & $88.2 \pm 2.2$ & $29 \pm 13.4$ & 134 \\
\hline 70 & 3 & $83.4 \pm 2.1$ & $41 \pm 12.0$ & 120 & 3 & $92.4 \pm 2.5$ & $40 \pm 16.2$ & 130 \\
\hline 80 & 3 & $91.0 \pm 3.4$ & $42 \pm 5.5$ & 104 & 3 & $96.7 \pm 0.5$ & $34 \pm 10.7$ & 115 \\
\hline 100 & 2 & $98.6(98.5-98.7)$ & $34(26-4 I)$ & 101 & 2 & 98.1 (97.2-98.9) & $4 \mid(2 \mid-60)$ & 86 \\
\hline
\end{tabular}

\section{Emergence}

Irradiation of 20-26 hr old pupae had no effect on adult emergence. Overall, emergence was high (Fig. 2), on average $96 \pm 0.6 \%$. No significant differences were observed between adult emergence for the different treatments compared to the control (independent t-tests, data not shown).

\section{Induced sterility}

The total number of eggs laid in all experiments was 78600 of which 46600 eggs were checked to estimate the proportion that had hatched. The average number of eggs laid per female in all treatments was similar to the control for both irradiated life stages (pupae: $\left(\mathrm{F}_{8,20}=0.65\right.$, $\mathrm{p}>0.05)$; adults: $\left(\mathrm{F}_{6,13}=0.74, \mathrm{p}>0.05\right)$, (Table 2).

Control sterility (i.e. the number of eggs that naturally do not hatch) in the KGB colony is $26 \pm 9 \%$. Corrected for this control sterility, the residual fertility decreased with increasing radiation dose for both pupal and adult stages (Fig. 3). A linear regression (pupae $\left(\mathrm{F}_{1,20}=105.3, \mathrm{p}<0.01\right.$; adults $\left.\left(\mathrm{F}_{1,14}=142.3, \mathrm{p}<0.01\right)\right)$ was obtained after transformation of both axes, and the regression model can be used to predict induced sterility rates (= 1 - residual fertility) at specific irradiation doses.

For the pupal stage the equation is:

N.E.D. (residual fertility) $=6.07-3.95\left(\log _{10}(\right.$ dose $/$ Gy $)$ ) with $\mathrm{r}^{2}=84 \%$;

for the adults:

N.E.D. (residual fertility) $=6.33-4.22\left(\log _{10}(\right.$ dose $/$ Gy $\left.)\right)$ with $\mathrm{r}^{2}=91 \%$.

Plotting fertility on a logarithmic scale against dose provides insight in the nature of dominant lethal mutations. A linear response indicates a "one-hit" relationship whereas departures from linearity indicate a "multi-hit" relationship $[27,28]$. Graphs for the pupal and adult stage show a predominantly linear relationship (Fig. 3) suggesting a one-hit relationship to dose; i.e. a large proportion of dominant lethal mutations result from single events in the gametes. As expected, at higher doses the lines tend to depart from linearity suggesting that gametes carry more than one dominant lethal mutation.

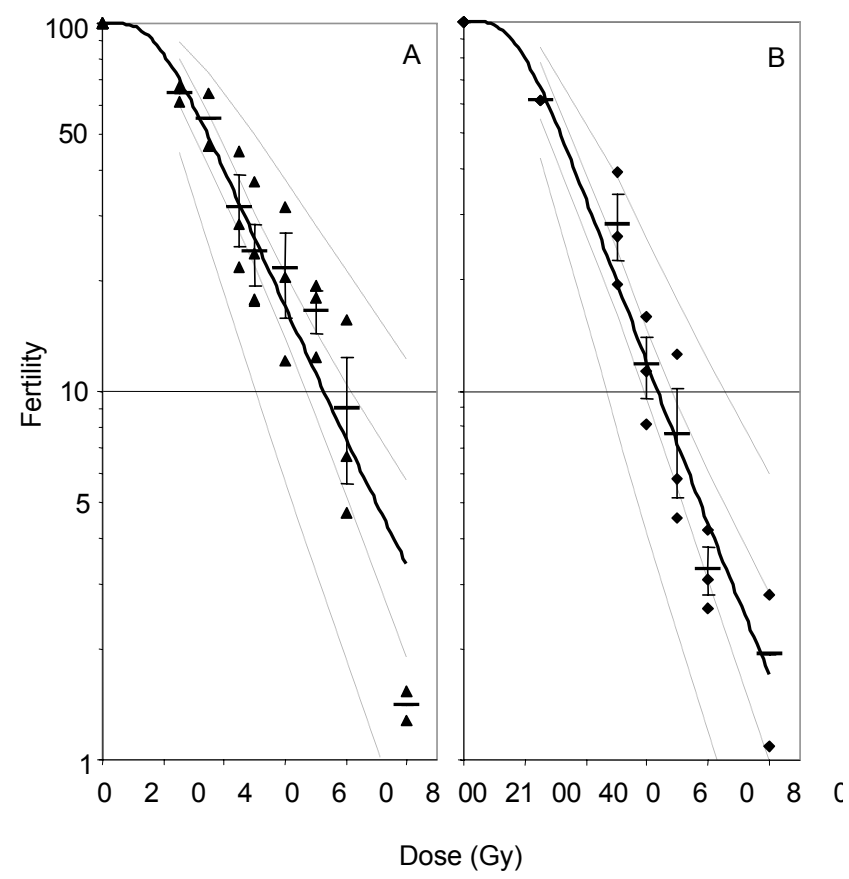

\section{Figure 3}

Regression line $( \pm 95 \% \mathrm{Cl}$ for mean and individual values) for fertility versus irradiation dose (Gy) for male An. arabiensis pupae (A) and adults (B). Symbols indicate observed individual values. Means $( \pm S E$, if $n>2)$ are indicated by a horizontal line. 
When comparing the two stages, germ cells of adults were more susceptible to irradiation resulting in higher induced sterility levels (GLM: Stage $\mathrm{F}_{1,24}=4.55, \mathrm{p}<0.05$ ) (Fig. 4). More specifically, irradiation of adults yielded higher sterility than irradiation of pupae at doses between 60 and 80 Gy (Table 2) although no significant differences were observed between the two stages for each dose individually (independent t-tests, data not shown).

\section{Mosquito survival}

The recovery of adults after irradiation was $100 \%$. In total, survival was scored for 2303 males. Because the series controls were significantly different in the pupal (Log-rank = 18.76, $\mathrm{df}=6, \mathrm{p} \leq 0.01$ ), and adult stage (Log-rank = 13.1, $\mathrm{df}=3, \mathrm{p} \leq 0.01$ ), survival curves were analysed per series against the control. For the pupal stage, the survival curve for irradiated males was not significantly lower than the control, except in series 1 where males irradiated with 25 and 35 Gy had lower survival (Table 1). Higher survival was also found in certain treatments. In the adult stage, survival of irradiated males was similar to the control, with the exception of 70 Gy males in series 1, for which survival was higher (Table 1).

\section{Insemination}

A total number of 999 females was dissected (on average $21.7 \pm 0.6$ females per cage) and examined for insemination (except in series 1 for the pupal stage; no dissections were performed). After on average ten days of mating, control insemination was $79 \% \pm 4$ for females confined with males irradiated at the pupal stage, and $89 \% \pm 4$ in for those with males irradiated in the adult stage, and no significant differences were observed between both controls $\left(\mathrm{t}_{\mathrm{s}}=0.40, \mathrm{df}=8, \mathrm{p}=>0.05\right)$. For the pupae, a significant negative correlation $(\mathrm{r}=-0.47, \mathrm{p}<0.01)$ was found between dose and insemination (Fig. 5). For adults, no significant correlation was observed $(\mathrm{r}=-0.37, \mathrm{p}>0.05)$. Individual t-tests showed that insemination rates were not statistically different from the control for all treatments in both stages, except at $100 \mathrm{~Gy}$ for the pupal stage $\left(t_{s}=3.11\right.$, $\mathrm{df}=6, \mathrm{p}<0.05)$. However, when comparing the two stages at individual doses, no significant differences were found (independent t-tests, data not shown). A positive correlation was found between fecundity (number of eggs/female) and insemination of females placed together with males irradiated in the pupal stage $(\mathrm{r}=0.67, \mathrm{p}<$ $0.01),\left(r^{2}=45 \%\right)$.

\section{Discussion}

Ionizing radiation has, over the years, proven to be a safe and reliable way to induce sterility in a large variety of insects [2]. The potential use of the SIT against malaria vectors is currently explored and the development of radiation sterilisation protocols is a vital part of such endeavour.

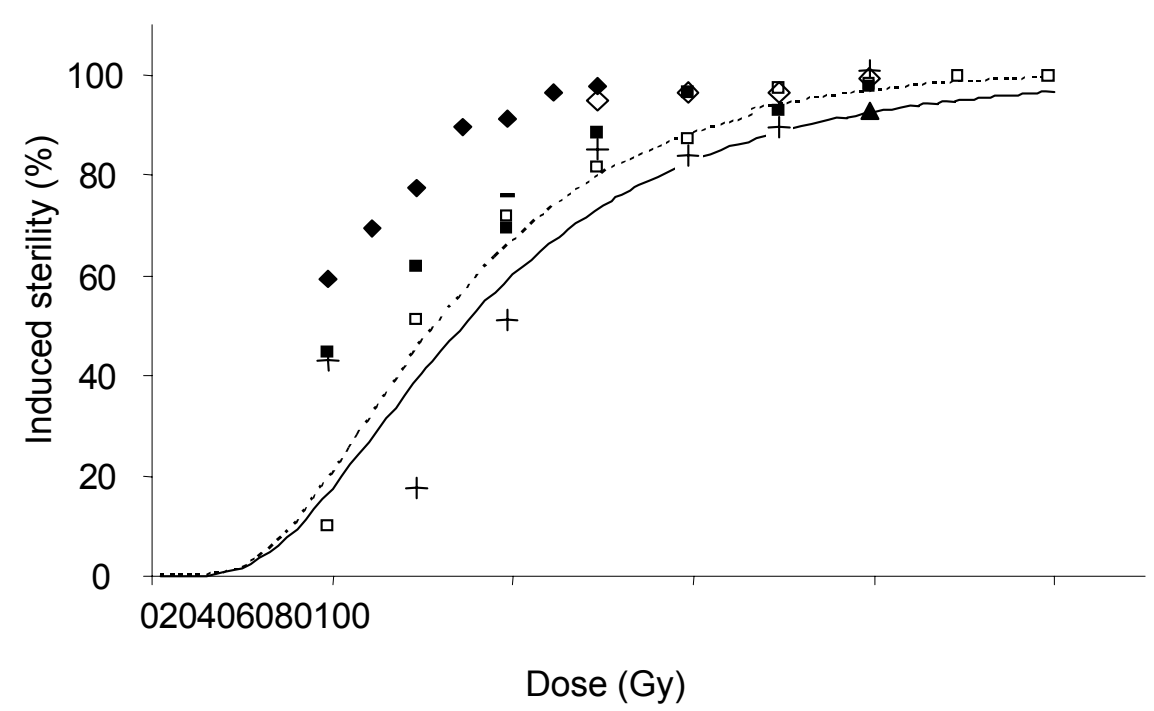

\section{Figure 4}

Comparative analysis of dose-sterility data from this study (solid line for An. arabiensis pupae, dotted line for adults) and published reports on anopheline irradiation (using 20-100 Gy). Induced sterility levels were calculated from observed sterility levels for [7,18]. +:An. albimanus pupa [17]; $\square$ : An. stephensi pupa [7]; $\square$ : An. stephensi adult [20]; $\diamond:$ An. pharoensis pupa [18]; $\diamond:$ An. pharoensis adult [19]; $\mathbf{\Delta}$ : An. gambiae adult [22]; -An. arabiensis adult [2I]. 


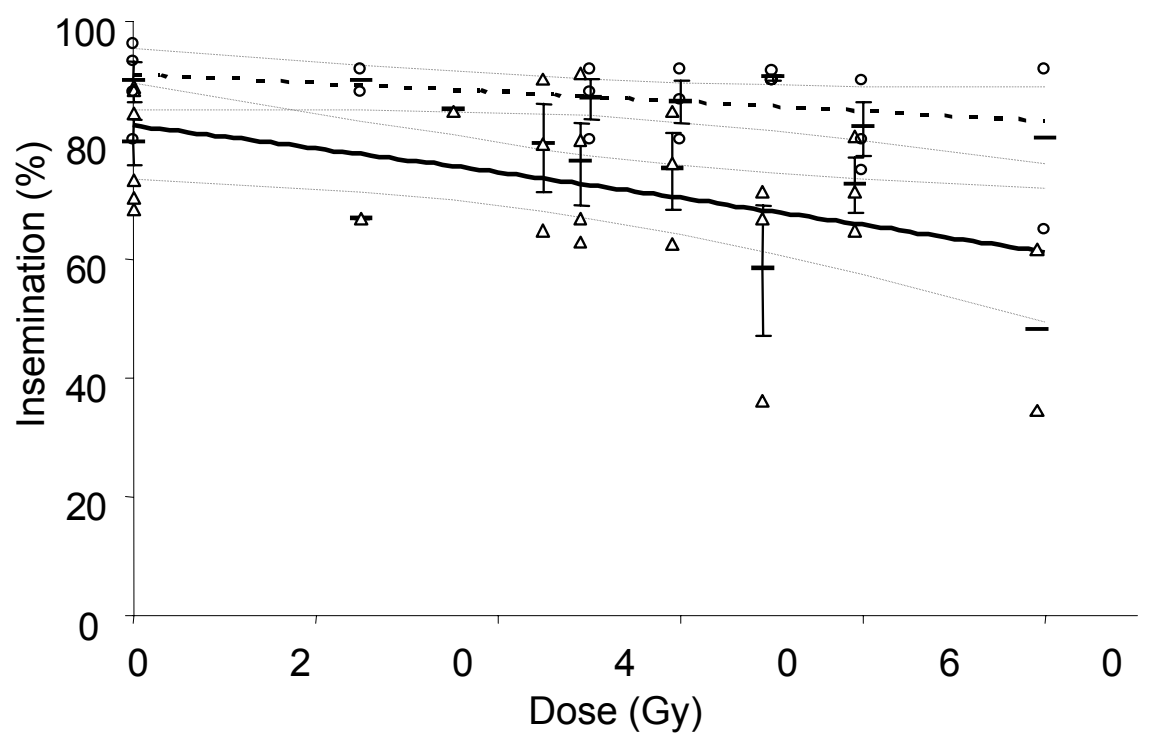

\section{Figure 5}

Insemination of virgin females mated with An. arabiensis males irradiated in pupal or adult stage for the different treatments. Triangles represent pupal individual values, circles adult individual values. Detransformed means $( \pm S E$ if $n>2)$ are indicated by a horizontal line. Linear regression lines $( \pm 95 \% \mathrm{Cl})$ are given; solid line is pupae, dotted line adults. For clarity purposes, data points for pupae have been shifted slightly to the left.

Most experimental work on Anopheles irradiation has focused on the pupal stage $[7,17,18,25,29]$. The irradiation of pupae is preferably performed on older ( $>15 \mathrm{hrs}$ ) pupae since irradiation of young pupae results in a reduced emergence [22,25] and shorter survival [30]. In this study, age of the pupae when irradiated was between 22-26 hrs and 7-12 hrs before emergence. We found that the irradiation had no effect on the emergence from the pupae even when the dose applied was high. Similar results were reported for pupal irradiation of An. pharoensis [25].

Although irradiation is intended to target the germ cells, the process is non-specific and somatic cells may also be damaged. One of the commonest effects of somatic damage is a reduced longevity [31]. In this study, males irradiated as pupae or adults with increasing doses had similar or even higher survival times compared to the controls. Only in two cases a reduced survival was found, but as this was observed at the lowest doses, irradiation is not the likely cause. For An. pharoensis [32], a slight increase in longevity of males irradiated with 5-70 Gy as pupae compared to the control was reported. Other studies report a reduced longevity; in An. pharoensis [25] for pupae irradiated at 100-130 Gy, and in A. stephensi [7] for pupae irradiated at 80 and 120 Gy. Sampling of mosquitoes occurred at 48-72 hrs intervals over the weekends, which could have influenced consistency of the data to some extent. However, as the controls were exposed to the same treatment and subsequent analyses we deem this variation caused by different sampling intervals of negligible importance.

The fecundity of the females mated with irradiated males was similar for all treatments compared to the control. Due to lower insemination of females placed with males irradiated at higher doses in the pupal stage, a positive correlation was observed between fecundity and insemination rate. Overall, fecundity rates were variable because eggs were collected en masse and fecundity calculated over all females alive regardless if they had blood fed or oviposited. Differences observed in fecundity are partly accounted for by the reduced insemination rate found in certain treatments.

Unlike in some other mosquito colonies, uninseminated females of the KGB strain used in these experiments have not been observed to oviposit, resulting in fewer eggs when the insemination rate is low. Other, inexplicable variation in egg batch sizes have been observed, yet should not influence our conclusions since these occurred throughout the experimental period and across all treatments.

The dose-sterility curves in An. arabiensis for pupae and adults show the classic pattern found for such curves of a 
linear relationship at low doses and the flattening of the curve at higher doses $[33,34]$. The highest dose of $100 \mathrm{~Gy}$ induced $>98 \%$ sterility. Overall, we found that fertility is slightly more sensitive to exposure in the adult stage than in the pupal stage. There are only a few studies with anophelines that have irradiated both stages simultaneously. At $120 \mathrm{~Gy}$, equal levels of sterility were found for pupal and adult irradiation in An. gambiae and $A n$. stephensi [23]; while in An. gambiae pupae were more radiation-resistant than adults [22]. If we compare levels of sterility found in different studies, An. stephensi [20] and An. pharoensis [19] both show higher sterility levels in the adult stage (Fig. 4).

When comparing the level of induced sterility in An. arabiensis with other anophelines (Fig. 4), An. arabiensis shows a greater radiation resistance resulting in lower induced sterility levels. In the pupal stage, An. albimanus [17] behaved similarly at doses > $40 \mathrm{~Gy}$, while An. stephensi [7] and An. pharoensis [18] overall had slightly higher induced sterility levels. In the adult stage, $A n$. pharoensis [19] and especially An. stephensi [20] showed higher sterility levels. Only in An. gambiae [22] at 80 Gy a lower level of induced sterility was observed. In a study in which adult An. arabiensis were irradiated with 40 Gy [21], a somewhat higher level of sterility was reported, but the causes for this remain unclear.

Although competitiveness experiments will be used to assess male fitness at different radiation doses, the level of insemination in the absence of competition gives some indication of the male's ability to mate. We found a substantial variation in insemination within some treatments, but the number of replicates was low. The insemination of females mated with males irradiated as adults was comparable to the control. For males irradiated at the pupal stage, a negative correlation was found between insemination and dose but the correlation-coefficient $\left(\mathrm{r}^{2}\right)$ was associated with less than $<25 \%$ of the total variation and only the dose of 70 Gy was different to the control. When the two stages were compared at individual doses, no differences were observed. In previous studies, equal insemination [17] or similar egg production $[18,25]$ in females mated to males irradiated as pupae was observed. The results in this study suggest a decline in mating ability with increasing dose in males irradiated as pupae, but not as adults.

\section{Conclusion}

In the past, SIT focused on the induction of almost 100\% sterility and this led to the use of high radiation doses. Over the years, it was observed that some insect species irradiated with these high doses were not able to suppress the natural population due to a lack of competitiveness. A revision of requiring $100 \%$ sterility was needed and it is suggested that more sterility can be induced in the target population if insects are more competitive when subjected to semi-sterilising doses $[2,35]$. Male competitiveness at these lower doses needs to be estimated in a semifield setting, where irradiated males compete with nonirradiated wild males for wild females. On the basis of such experiments, the optimal dose for the released male mosquitoes can be identified. On the other hand, reduced competitiveness can be compensated for by increasing the flooding ratio of sterile males in the field [36].

The choice of developmental stage for irradiation in an SIT programme depends on numerous factors including handling, survival, sterility, competitiveness and release methodology and this study has focused on the first three factors. The mosquitoes in this study were irradiated in small numbers and both stages survived the handling and irradiation process well. Up-scaling the irradiation process for mass production remains a challenge. Pupal irradiation has a number of advantages over adults but not enough resources have been directed to the development of large-scale irradiation devices to draw conclusions.

The longevity of irradiated males was similar to the controls in the adult stage. In the pupal stage, overall similar or higher survival was observed compared to the control. The possibility that irradiation has a beneficial effect on longevity in the pupal stage cannot be excluded from these results, yet longevity was not measured under stressful conditions. In the Mediterranean fruit fly Ceratitis capitata (Wiedemann), it is known that under stress the possible negative effects of radiation tend to become more pronounced. Quality control testing in Mediterranean fruit fly SIT programmes measures longevity after the deprivation of food for some time [37]. Although mosquitoes are more sensitive to complete food deprivation, similar tests can be devised for mosquitoes to assess the impact of irradiation.

In this study, fertility was slightly more sensitive to irradiation exposure in the adult stage than in the pupal stage, but differences were small. The trend to reduced insemination rates at higher doses in the pupal stage suggests that pupae are more somatically damaged by the irradiation process than adults, a finding supported by studies that observed that pupae irradiation reduces competitiveness more so than adult irradiation $[22,23]$. Competitiveness of pupae irradiated at semi-sterilising doses has hardly been studied; but in An. stephensi it was observed that males irradiated as pupae with 80 Gy were 1.7 times more competitive than males irradiated with 120 Gy [7]. Future studies will focus on competition experiments that will include the use of sterilizing and semi-sterilizing doses for both developmental stages. 


\section{Authors' contributions}

MEHH planned and conducted the experiments, analysed the data and wrote the first draft of the paper. AP provided expertise on insect irradiation and contributed to the final draft of the paper, and BGJK supervised the work and contributed significantly to the final draft of the paper.

\section{Acknowledgements}

The authors would like to thank Ms G. Germershausen for dedicated laboratory assistance, Dr. A. Odulaja provided valuable guidance with the statistical analyses and we thank Dr. A. Robinson and the two anonymous reviewers for their comments, which significantly improved the quality of the manuscript.

\section{References}

I. UNICEF, WHO/RBM: World Malaria Report. WHO/HTMIMALI 2005. II 022005.

2. Dyck A, Hendrichs J, Robinson AS: The Sterile Insect Technique: Principles and Practice in Area-Wide Integrated Pest Management Heidelberg, Germany: Springer; 2005.

3. Benedict MQ, Robinson AS: The first releases of transgenic mosquitoes: an argument for the sterile insect technique. Trends Parasitol 2003, 19:349-355.

4. Dame DA, Lowe RE, Williamson DL: Assessment of Released Sterile Anopheles albimanus and Glossina morsitans morsitans. In Cytogenetics and genetics of vectors Edited by: Kitzmiller JB, Kanda T. Amsterdam, The Netherlands: Elsevier Biomedical; 1981:231-248.

5. IAEA: Development of the Sterile Insect Technique for mosquitoes. 2006 [http://www.iaea.org/programmes/naal/agri/ index.htm].

6. Helinski MEH, El-Sayed B, Knols BGJ: The Sterile Insect Technique: can established technology beat malaria? Entomol berichten 2006, 66: 13-20.

7. Sharma VP, Razdan RK, Ansari MA: Anopheles stephensi: effect of gamma-radiation and chemosterilants on the fertility and fitness for sterile male releases. J Econ Entomol 1978, 71:449-452.

8. Dame DA: Genetic control by sterilized mosquitoes. In Biological Control of Mosquitoes Volume 6. Edited by: Chapman R, Barr R, Weidhaas DE, Laird M. American Mosquito Control Association, Bull; 1985:159-172.

9. Alphey L: Re-engineering the sterile insect technique. Insect Biochem Mol Biol 2002, 32: 1243-1247.

10. Alphey L, Beard CB, Billingsley P, Coetzee M, Crisanti A, Curtis C, et al.: Malaria control with genetically manipulated insect vectors. Science 2002, 298: $119-121$.

1I. Thomas DD, Donnelly CA, Wood RJ, Alphey LS: Insect population control using a dominant, repressible, lethal genetic system. Science 2000, 287:2474-2476.

12. Knols BG], Louis C: Bridging Laboratory and Field Research for Genetic Control of Disease Vectors. In Proceedings of the joint WHO/TDR, NIAID, IAEA and Frontis Workshop on Bridging Laboratory and Field Research for Genetic Control of Disease Vectors. Nairobi, Kenya I416 July 2004 Wageningen. Frontis; 2005.

13. Scott TW, Takken W, Knols BG, Boete C: The ecology of genetically modified mosquitoes. Science 2002, 298: I17-119.

14. LaBrecque GC, Bowman WC, Patterson RS, Seawright JA: Persistence of thiotepa and tepa in pupae and adults of Culex fatigans. Bull World Health Organ 1972, 74:676.

15. Hayes W]: Toxicological aspects of chemosterilants. In Principles of insect chemosterilisation Edited by: LaBrecque GC, Smith CN. New York, USA: Appleton Century Crofts; 1968:315-347.

16. Bracken GK, Dondale CD: Fertility and survival of Achaearanea tepidariorum (Araneida:Theridiidae) on a diet of chemosterilized mosquitoes. Can Entomol 1972, 104:1709-17/2.

17. Ali SR, Rozenboom LE: Observations on sterilization of Anopheles (C.) albimanus Wiedemann by $\mathbf{X}$-irradiation. Mosq News 1972, 32:574-579.

18. Abdel-Malek AA, Wakid AM, Tantawy AO, El-Gazzar LM: Studies on factors influencing the induction of sterility in Anopheles pharoensis Theobald by gamma radiation. The use of isotopes and pesticides in pest control. Proceedings of a symposium held in BeirutLebanon, March 1974 1975:161-174.

19. Shoukry A: X-ray induced sterility, dominant lethality and inherited semisterility in Anopheles pharoensis Theo (Dipt., Culicidae). Z Angew Entomol 1980, 89:498-504.

20. Akram M, Aslamkhan M: Production of dominant lethal mutations by gamma-irradiation in the malaria mosquito, Anopheles stephensi. Pak J Zool 1975, 7:177-I84.

21. Lines JD, Curtis CF: Genetic sexing system in Anopheles arabiensis Patton (Diptera: Culicidae). J Econ Entomol 1985, 78:848-85I.

22. Curtis CF: Radiation sterilization. Report on mosquito research. Ross Institute of Tropical Hygiene. 01.01.76-3I.I2.77 1976.

23. Andreasen $\mathrm{MH}$, Curtis CF: Optimal life stages for radiation sterilization of Anopheles for sterile insect releases. Med Vet Entomol 2005, 19:238-244.

24. IAEA: Gafchromic Dosimetry system for SIT. 2004 [http:// www-naweb.iaea.org/nafa/ipc/public/Dosimetry SOP v10b.pdf].

25. Abdel-Malek AA, Tantawy AO, Wakid AM: Studies on the eradication of Anopheles pharoensis Theobald by the Sterile-Male technique using cobalt-60. III. Determination of the Sterile Dose and its biological effects on different characters related to "Fitness" components. J Econ Entomol 1967, 60:20-23.

26. Abbott WS: A method of computing the effectiveness of an insecticide. J Econ Entomol 1925, 18:267.

27. LaChance LE: The induction of dominant lethal mutations in insects by ionizing radiation and chemicals - as related to the sterile-male technique of insect control. In Genetics of insect vectors of disease Edited by: Wright JW, Pal R. Amsterdam, The Netherlands: Elsevier; 1967:617-650.

28. Curtis CF: Induced sterility in insects. Adv Reprod Physiol 1971, 5:120-165.

29. Tantawy AO, Abdel-Malek AA, Wakid AM: Studies on the eradication of Anopheles pharoensis Theobald by the Sterile-Male technique using cobalt-60. V. Mating competitiveness of radiosterilized males. J Econ Entomol 1967, 60:696-699.

30. Davis AN, Gahan JB, Weidhaas DE, Smith CN: Exploratory studies on gamma irradiation for the sterilization and control of Anopheles quadrimaculatus. J Econ Entomol 1959, 52:868-870.

31. Proverbs MD: Induced sterilization and control in insects. Annu Rev Entomol 1969, 14:8I-102.

32. Abdel-Malek AA, Tantawy AO, Wakid AM: Studies on the eradiaction of Anopheles pharoensis Theobald by the Sterile-Male technique using cobalt-60. I.Biological effects of gamma radiation on the different developmental stages. J Econ Entomol 1966, 59:672-678.

33. LaChance LE, Graham CK: Insect radiosensitivity: dose curves and dose-fractionation studies of dominant lethal mutations in the mature sperm of $\mathbf{4}$ insect species. Mutat Res 1984, 1 27:49-59.

34. Robinson AS: Mutations and their use in insect control. Mutat Res 2002, 5 I I: I I3-I32.

35. Toledo J, Rull J, Oropeza A, Hernández E, Liedo P: Irradiation of Anastrepha obliqua (Diptera: Tephritidae) revisited: Optimizing sterility induction. J Econ Entomol 2004, 97:383-389.

36. Knipling EF: Possibilities of insect population control through the use of sexually sterile males. J Econ Entomol 1955, 48:459-462.

37. FAO/IAEA/USDA: Manual for Product Quality Control and Shipping Procedures for Sterile Mass-Reared Tephritid Fruit Flies, Version 5.0. Vienna, Austria, International Atomic Energy Agency; 2003:85. 